\title{
Ergotherapie für Pferde
}

\author{
Yvonne Katzenberger, Ruth Katzenberger-Schmelcher
}

Vielen Menschen wird die Ergotherapie als Begriff geläufig sein, weil sie schon selbst in ergotherapeutischer Behandlung waren. Jedoch ist den wenigsten bewusst, was Ergotherapie genau heißt und vor allem, warum es sinnvoll ist, Ergotherapie als Therapieform auch beim Pferd einzusetzen. Im Folgenden ein kurzer Einblick.

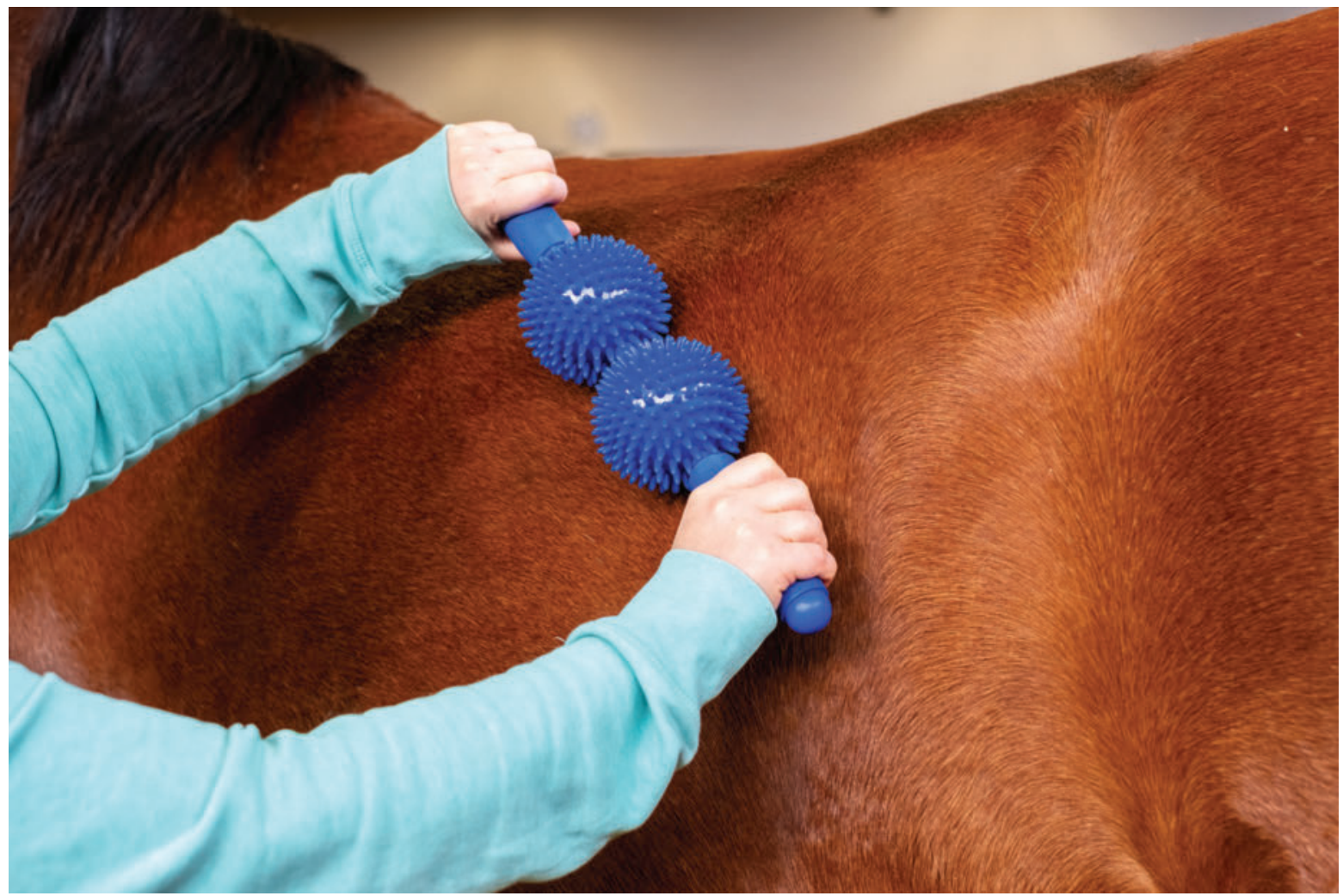

Stimulierung des taktilen Systems mit dem Igelballroller @ Kirsten Oborny (Katzenberger/Katzenberger-Schmelcher, Ergotherapie für Pferde, 2019, Thieme)

\section{Ergotherapie für Pferde - eine Begriffsbestimmung}

Der Begriff der Ergotherapie stammt aus dem Griechischen und bedeutet soviel wie Gesundung durch Handeln und Arbeiten. Im Humanbereich gehört die Ergotherapie zu den medizinischen Heilberufen. Sie ist dort bereits seit Jahrzehnten fester Bestandteil des therapeutischen Spektrums und wird erfolgreich als anerkanntes Heilmittel eingesetzt. Die Ergotherapie geht von einem ganzheitlichen Ansatz aus. Als Therapieform verfolgt sie vor allem das Ziel, die Handlungsfähigkeit des Menschen im Alltagsleben präventiv zu fördern oder wiederherzustellen.

In gewissem Rahmen und mit einigen Modifikationen können die Prinzipien der Ergotherapie auch auf das Pferd übertragen werden: 


\section{DEFINITION}

Ergotherapie für Pferde oder Pferdeergotherapie unterstützt und begleitet Pferde jeden Alters und jeder Rasse, die in ihrer Handlungsfähigkeit eingeschränkt oder von Einschränkung bedroht sind. Ziel ist es, sie bei der Durchführung von für ihre Gesundheit bzw. für ihren Besitzer bedeutungsvoller Betätigungen im Pferdealltag zu stärken. Hierbei dienen spezifische Aktivitäten, Umweltanpassung und Beratungen des Besitzers dazu, dem Pferd die Handlungsfähigkeit in ihm vom Besitzer vorgegebenen Alltag und eine Verbesserung seiner Lebensqualität zu ermöglichen [1].

Es geht also darum, die Handlungsfähigkeit des Pferdes und damit den Pferdealltag zu verbessern. Während die Tierphysiotherapie eher einen funktionsorientierten Ansatz verfolgt, geht es bei der Ergotherapie um einen handlungsorientierten Ansatz.

Dabei ist besonders die Rolle des Pferdebesitzers zu beachten. Er muss eine sogenannte Compliance, also ein kooperatives Verhalten entwickeln, um die Probleme seines Pferdes akzeptieren, angehen und ressourcenorientiert handeln zu können. So wird auch der Umgang des Pferdebesitzers mit dem Pferd als Ausgangspunkt für Veränderungen genutzt. Schließlich liegt es in seiner Verantwortung und Verpflichtung, den Pferdealltag und die Betätigungsbereiche des Pferdes so zu gestalten, dass ein art- und tierschutzgerechtes Pferdeleben möglich ist. Deshalb eruiert der Therapeut in enger Kommunikation mit dem Pferdebesitzer, in welchen Lebens- und Alltagsbereichen des Pferdes Verbesserungen notwendig oder wünschenswert sind.

\section{Die Wahrnehmungsverarbeitung und die Basissinne beim Pferd}

Den Grundstein für die ergotherapeutische Intervention am Pferd bildet die sogenannte sensorische Integration. Die amerikanische Entwicklungspsychologin Jean Ayers hat diesen Begriff im Humanbereich definiert:

\section{DEFINITION}

Die sensorische Integration sortiert, ordnet und vereint alle sinnlichen Eindrücke des Individuums zu einer vollständigen und umfassenden Hirnfunktion [2].

Vereinfacht ausgedrückt bedeutet sensorische Integration soviel wie „Wahrnehmungsverarbeitung“. Die
Prinzipien der sensorischen Integration können auf Pferde übertragen werden: Alle Informationen, die über die Sinnessysteme - die sogenannten Basissinne - aufgenommen werden, werden integriert. Das heißt, sie werden im Nervensystem und Gehirn weitergeleitet, verarbeitet und gedeutet, sodass Pferde in bestimmten Situationen auf eine bestimmte Art und Weise handeln.

Die Existenz und das Zusammenwirken der Basissinne ermöglicht dem Pferd - über eine entsprechende Reizaufnahme-eine Empfindungs- und Wahrnehmungsfähigkeit. Hierbei gilt es zu beachten, dass der Organismus polymodal funktioniert, d. h. dass kein Basissinn isoliert von den anderen funktioniert. Die 3 Basissinne, das taktile, das propriozeptive und das vestibuläre System, sind somit stets in ihrem Zusammenwirken zu betrachten.

\section{Das taktile System}

Es ist als größtes Wahrnehmungssystem für die Wahrnehmung der Oberflächensensibilität verantwortlich und umfasst den Körper mit seiner gesamten Hautoberfläche: Die Haut schützt das Pferd als größtes Organ vor mechanischen, chemischen und thermischen Einflüssen. Sensoren, die über die gesamte Hautoberfläche verteilt sind, nehmen die verschiedenen Reize im Sinne der nervalen Informationsvermittlung auf und leiten sie weiter. Die Informationen der taktilen Wahrnehmung vermitteln dem Pferd Kenntnis von der Ausdehnung und den Grenzen des eigenen Körpers.

\section{Das propriozeptive System}

Das System ist für die Wahrnehmung der Tiefensensibilität verantwortlich. Mithilfe der Propriozeptoren wird die Stellung der Glieder zueinander sowie die Gliederbewegung und Muskelspannung registriert. Ebenso werden Bewegungen und Haltungen, die durch die eigene Bewegung entstehen, wahrgenommen. So wird es dem Pferdekörper ermöglicht, jederzeit die Stellung der Extremitäten zu erfassen und die Muskelkraft zu dosieren, mit der einzelne Körperteile bewegt werden.

\section{Das vestibuläre System}

Das vestibuläre System oder Gleichgewichtssystem sorgt für die Aufrechterhaltung des Körpers und die Orientierung im Raum. Das Vestibularorgan liegt im Innenohr am Felsenbein und bildet eine anatomische Einheit mit dem Hörorgan. Dort werden z.B. Drehbeschleunigungen, Richtungsänderungen und die Schwerkrafteinwirkung registriert. Das vestibuläre System ermöglicht geordnete Körperhaltungen und -bewegungen.

Die Wahrnehmungsverarbeitung sorgt somit dafür, dass das Pferd durch das Zusammenspiel der Basissinne ein hinreichendes Körperbewusstsein und eine entsprechende Körperwahrnehmung erlangt. Ist die Wahrnehmung über 
die Basissinne nicht adäquat möglich oder gibt es Defizite in der Wahrnehmungsverarbeitung, können sich gewisse Fähigkeiten des Pferdes wie etwa Balance, Koordination und Durchlässigkeit nicht im notwendigen Ausmaß entwickeln.

\section{Merke}

Diese Defizite äußern sich oftmals in Verhaltensweisen, die von vielen Pferdebesitzern gerne als „Unart“ fehlinterpretiert werden.

\section{Indikationen}

Indikationen für eine ergotherapeutische Behandlung des Pferdes sind variationsreich und können ganz unterschiedlich aussehen. Gemein ist ihnen allen, dass das Pferd in den aufgeführten Situationen einer Stimulierung der Basissinne ausgesetzt ist. Somit können alle Auffälligkeiten, die auf Defizite in der Wahrnehmung bzw. der Wahrnehmungsverarbeitung des Pferdes schließen lassen können, als Behandlungsindikationen gewertet werden.

\section{INDIKATIONEN}

Der Pferdealltag ist breit gefächert und kann in verschiedene Alltagssituationen gegliedert werden. Beispiele für Indikationen sind Auffälligkeiten ...

- im Stall: Das Pferd hat häufig kleinere, oberflächliche Verletzungen und Schürfwunden an der Haut, weil es an Wänden, Türen, Futterraufen etc. anstößt.

- im täglichen Umgang: Das Pferd lässt sich nicht oder nur schwer eindecken oder beißt nach der Decke.

- bei der Bodenarbeit: Beim Übertreten von Stangen stößt das Pferd sehr häufig mit den Hufen an die Stangen.

- beim Reiten: Sobald der Reiter Seitengänge abrufen will, zeigt das Pferd Koordinationsprobleme und/oder widersetzt sich den Hilfen.

- beim Tierarzt: Das Pferd lässt sich keine Wurmkur ins Pferdemaul verabreichen, weil es den Kopf nach oben reißt oder steigt.

\section{Übungen zur Schulung der Basissinne}

In der Praxis muss der Therapeut eine therapeutische Situation und Reizangebote schaffen, die eine gezielte Schulung der Wahrnehmung ermöglichen. Dabei soll es weder zu einer Reizüberflutung noch zu einer monotonen Stimulation kommen. Der Therapeut muss also mithilfe von verschiedenen Therapiemedien, die schwerpunktmäßig einem Basissinn zugeordnet sind, die Wahrnehmungsverarbeitung des
Pferdes fördern und dabei stets überprüfen, ob die Übung in der Ausführung, Dauer oder Schwierigkeit anzupassen ist.

Merke

Der Therapeut muss das Pferd präzise beobachten, um das therapeutische Setting stets adaptieren zu können.

\section{Stimulationsschwerpunkt taktiles System: Kombination Igelballroller und Balance-Pad- Klopfer}

Material

Ein Igelballroller und ein Balance Pad.

\section{Ausführung}

Der Therapeut rollt das Pferd zunächst mit dem Igelballroller mit moderatem Druck und mit dem Strich des Pferdefells ab, wobei knöcherne Stellen (vor allem die Wirbelsäule) ausgespart werden. Die meisten Pferde sind es gewohnt, an der Schulter berührt zu werden, insofern bietet es sich an, dort zu beginnen. Das Abrollen erfolgt rhythmisch und für 2 Minuten ( $\triangleright$ Abb. 1).

Im Anschluss wird das Balance Pad auf der Schulter aufgelegt und der Therapeut klopft mit moderatem Druck für 2 Minuten mit den flachen Händen rhythmisch auf das Pad. So arbeitet er sich bis zur Kruppe vor. Im Anschluss wird das Pferd wiederum für 2 Minuten mit dem Igelballroller abgerollt ( $\bullet$ Abb. 2).

\section{Funktion}

Beim Abrollen mit dem Igelballroller werden hauptsächlich die sich in der Haut befindlichen Merkelzellen und Ruffini-Körperchen stimuliert. Durch das Abrollen werden auch die Vater-Pacini-Körperchen angeregt. Der Druck wird beim Abrollen großflächiger gegeben,

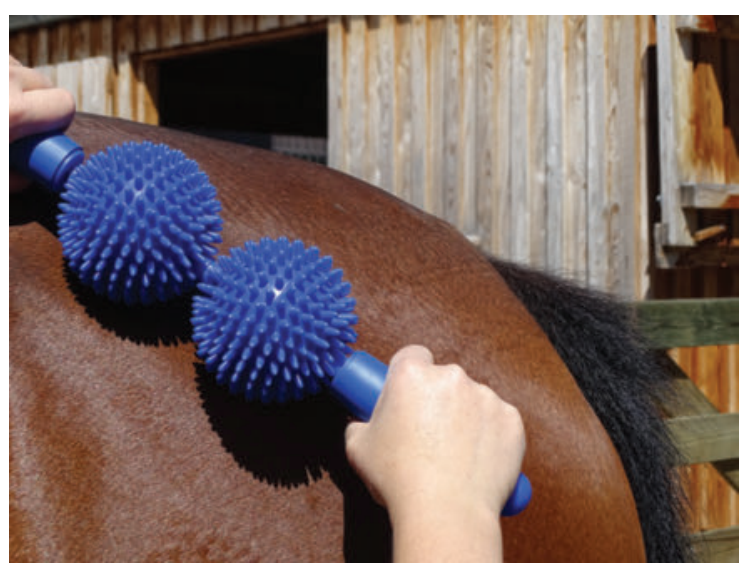

- Abb. 1 Der Therapeut rollt mit moderatem Druck in Fellrichtung. Knöcherne Stellen werden nicht abgerollt. (c) Yvonne Katzenberger, Ruth Katzenberger-Schmelcher 


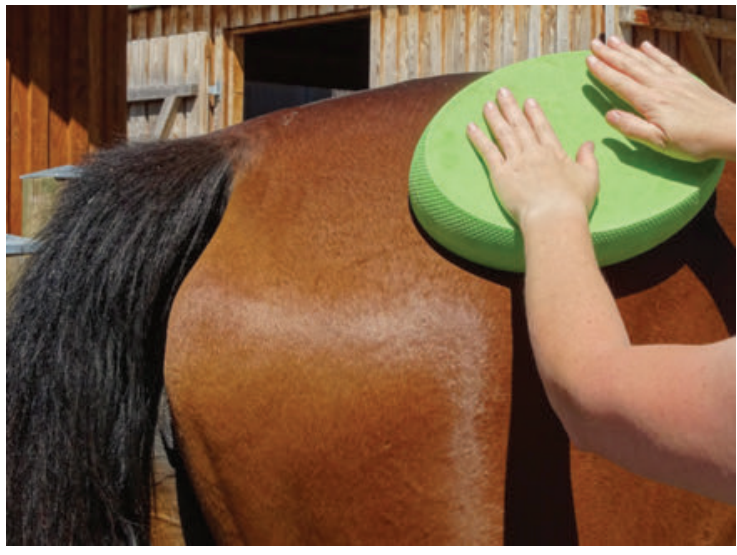

- Abb.2 Der Therapeut klopft mit den flachen Händen rhythmisch auf das Balance Pad. ( ) Yvonne Katzenberger, Ruth Katzenberger-Schmelcher

wohingegen er beim Abklopfen mit dem Balance Pad diffuser und auf kleineren Flächen gesetzt wird.

\section{EXKURS}

Merkelzellen, Ruffini- und Vater-Pacini-Körperchen zählen zu den Mechanorezeptoren, die der Wahrnehmung mechanischer Reize dienen [1].

Sie werden funktionell klassifiziert und in folgende Reaktionsgruppen eingeteilt:

- SA (slowly adapting):

- SA-I (slowly adapting 1): kleines rezeptives Feld, spricht auf Intensität des Hautreizes an

- SA-II (slowly adapting 2): großes rezeptives Feld

- RA (rapidly adapting): kleines rezeptives Feld

- PC (pacinian corpuscle): großes rezeptives Feld

Die Lage der Rezeptoren in der Haut und die Innervationsdichte, d. h. die Zahl der afferenten Fasern $/ \mathrm{cm}^{2}$ in der Haut, bestimmen die Größe der rezeptiven Felder.

Die SA-I-Rezeptoren adaptieren langsam, sie bilden auf einen lang andauernden Reiz kontinuierlich Aktionspotenziale. Hierzu zählen die Merkelzellen, die in der Epidermis und in den Sinushaaren vorkommen und hauptsächlich auf Druck und Zugreize reagieren.

Die SA-II-Rezeptoren, die als Ruffini-Körperchen beschrieben werden und sich in der Dermis befinden, adaptieren hingegen langsam auf Reize und reagieren hauptsächlich auf Dehnung der Haut. Die Vater-Pacini-Körperchen (PC) sind rasch adaptierende Mechanorezeptoren in den tieferen Schichten der Haut, die besonders gut Vibrationsempfindungen vermitteln.

\section{Anwendungsbereiche}

Diese Übung hilft Pferden, ihre Körpergrenzen besser einschätzen zu können. So legen sich Pferde beim Wälzen seltener fest oder zeigen seltener kleine, oberflächliche Hautverletzungen. Auch beim Sattel- oder Gurtzwang kann diese Übung sinnvoll sein.

\section{Stimulationsschwerpunkt propriozeptives System: die Raute}

Material

Sie benötigen 4 Bodenstangen (Länge $3 \mathrm{~m}$ ) und 8 Pylonen. Die Bodenstangen werden als Raute gelegt, jeweils 2 Pylonen werden mittig an 1 Stange gestellt, sodass zwischen den Pylonen ca. $50 \mathrm{~cm}$ Abstand bestehen. In der Mitte der Raute wird eine Gymnastikmatte aus Qualitätsschaumstoff ausgelegt ( $\bullet$ Abb. $\mathbf{3}$ ).

\section{Ausführung}

Der Therapeut führt das Pferd am lockeren Strick im Schritt durch die Pylonen über die Bodenstange. Dabei zergliedert er die Bewegung so, dass das Pferd für jeweils 5 Sekunden angehalten wird, sobald 1 Vorderbein, 2 Vorderbeine und 1 Hinterbein über die Stange gesetzt wurden. Anschließend führt er das Pferd über die Matte, hält es für jeweils 10 Sekunden an, sobald beide Vorderhufe und anschließend alle 4 Hufe auf die Matte gesetzt wurden. Danach führt er das Pferd ohne Unterbrechung über die gegenüberliegende Stange. Nun geht er in einer großzügigen Volte eine der Bodenstangen an, die das Pferd noch nicht überstiegen hat und wiederholt die Übung mindestens 6-mal ( $\bullet$ Abb. 4).

\section{Funktion}

Die kinästhetische Differenzierungs-, Kopplungs-, Balanceund Reaktionsfähigkeit werden geschult. Die Rautenform

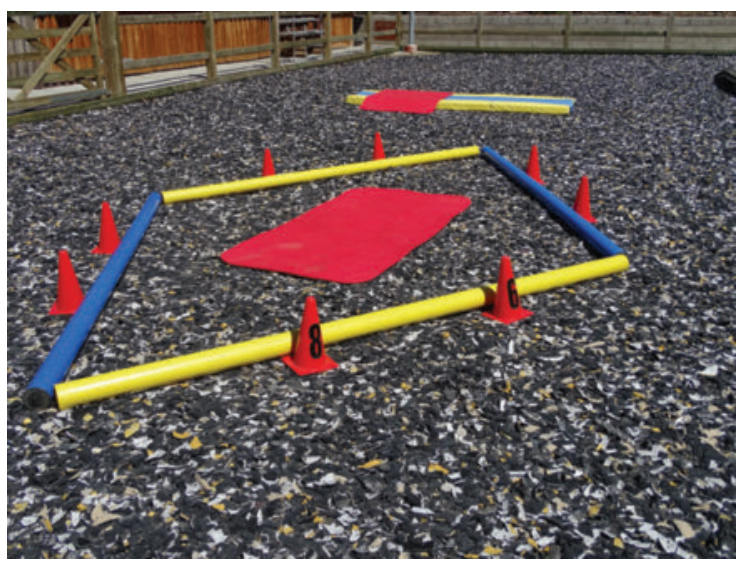

- Abb. 3 Die Stangen werden in Form einer Raute gelegt, die Gymnastikmatte befindet sich in der Mitte der Raute. c) Yvonne Katzenberger, Ruth Katzenberger-Schmelcher 


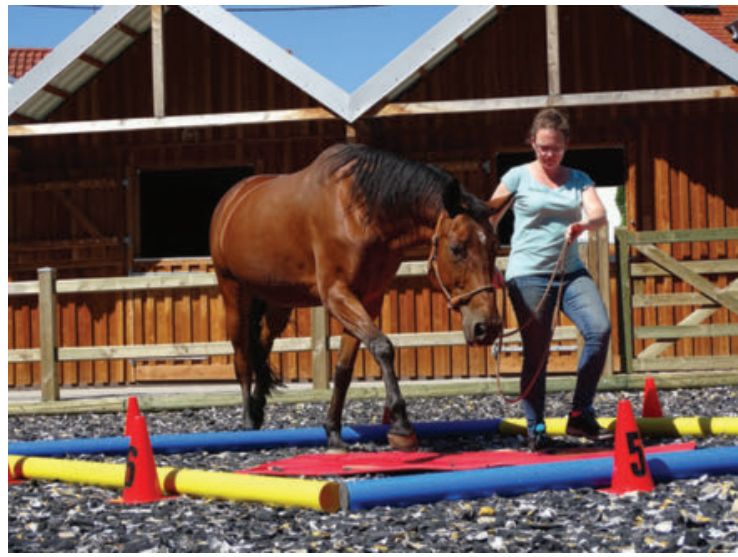

- Abb. 4 Das Pferd wird am lockeren Strick geführt, sodass es die Möglichkeit hat, den Kopf ohne Einwirkung gerade zu halten. () Yvonne Katzenberger, Ruth Katzenberger-Schmelcher

sorgt dafür, dass die Stangen in unterschiedlichen Winkelungen überstiegen werden müssen.

\section{Anwendungsbereiche}

Diese Übung empfiehlt sich insbesondere bei Pferden, die häufig an Bodenstangen anstoßen, egal ob mit oder ohne Reiter. Sie ist auch geeignet für Pferde, denen es schwerfällt innerhalb einer Gangart die Geschwindigkeit zu variieren. Durch diese Übung wird ein gezieltes Auffußen der Pferdebeine angebahnt. Die exakt abgestimmten Teilkörperbewegungen des Pferdes müssen sich stets einer veränderten Unterstützungsfläche anpassen. Das Laufen über einen forminstabilen Untergrund (die Matte) fördert die Balancefähigkeit und die Umstellungsfähigkeit.

\section{Stimulationsschwerpunkt vestibuläres System: die Schaumstoffgassenbrücke}

Material

Sie benötigen 3 Schaumstoffgassen und 1 Gymnastikmatte. Die 3 Gassen werden aneinander und die Matte darübergelegt.

\section{Ausführung}

Der Therapeut führt das Pferd im Schritt am lockeren Strick über die Gymnastikmatte. Die Übung wird 8-mal wiederholt ( $\bullet$ Abb.5).

\section{Funktion}

Durch Betreten des forminstabilen und stark nachgebenden Untergrunds erhält das Vestibularorgan vermehrt Reize aus der Peripherie. So werden auch unbekannte, nicht abgespeicherte Vestibularreize adäquat in das Bewegungsrepertoir integriert. Durch die natürliche Schrittfolge werden Reize des gewohnten Untergrunds (Reitplatzboden) mit Reizen des

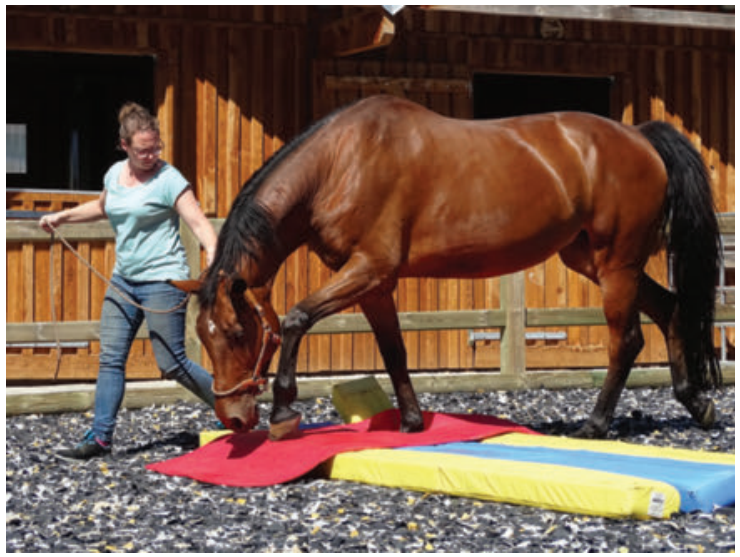

- Abb. 5 Die über die Gassen gelegte Gymnastikmatte sorgt für einen forminstabilen und stark nachgebenden Untergrund. ( ) Yvonne Katzenberger, Ruth KatzenbergerSchmelcher

ungewohnten Untergrunds (Schaumstoffgassenbrücke) kombiniert. Diese vestibulären Reize müssen entsprechend verarbeitet und integriert werden.

\section{Anwendungsbereiche}

Diese Übung eignet sich vor allem für Pferde, die taktunklar laufen und viel stolpern. Auch Pferde, die im Gelände Schwierigkeiten mit unterschiedlichen Bodenverhältnissen haben oder die sich beim Longieren und unter dem Sattel besonders unausbalanciert zeigen, profitieren von dieser Übung.

\section{Zusammengefasst für die Praxis}

Die Basissinne des Pferdes und damit die Fähigkeit zur Körperwahrnehmung können gezielt gefördert werden. Ergotherapeutische Interventionen können damit andere Therapieformen zielführend ergänzen und insbesondere dem Pferdebesitzer einen neuen Zugang zu seinem Pferd eröffnen.

\section{BUCHTIPP}

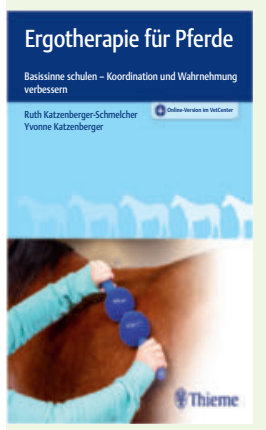
Ergotherapie für Pferde Basissinne schulen - Koordination und Wahrnehmung verbessern Ruth Katzenberger-Schmelcher, Yvonne Katzenberger 2019, ca. 190 Seiten, 200 Abbildungen, Georg Thieme Verlag KG ISBN Buch 978-3-13-242872-0 /ISBN ePub 978-3-13-2428274-4 /ca. $59,99 €$ 


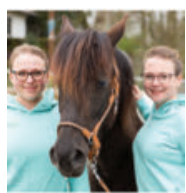

\section{Yvonne Katzenberger}

Staatlich geprüfte Ergotherapeutin; zertifizierte Reit- und Lerntherapeutin; seit 2004 eigene Praxis für Ergo- und Reittherapie mit therapeutischem Pferdestall; seit 2017 PFERGO (1. Akademie für Pferdeergotherapie); info@pfergo-akademie.de

\section{Dr. Ruth Katzenberger-Schmelcher}

Reittherapeutische Assistentin; passionierte Pferdetrainerin; seit 2017 PFERGO (1. Akademie für Pferdeergotherapie); info@pfergo-akademie.de

[1] Katzenberger-Schmelcher R, Katzenberger Y. Ergotherapie für Pferde. Stuttgart: Thieme; 2019

[2] Ayers AJ. Bausteine der kindlichen Entwicklung: Die Bedeutung der Integration der Sinne für die Entwicklung des Kindes. 4. Aufl. Berlin: Springer; 2002

\section{Bibliografie}

DOI https://doi.org/10.1055/a-0961-5767

Hands on 2019; 1: 45-50

(C) Georg Thieme Verlag KG Stuttgart · New York ISSN 2628-6033 